\title{
Regeneration Through in vivo Cell Fate Reprogramming for Neural Repair
}

\author{
Wenjiao Tai ${ }^{1,2}$, Xiao-Ming Xu ${ }^{3,4}$ and Chun-Li Zhang ${ }^{1,2 *}$ \\ ${ }^{1}$ Department of Molecular Biology, University of Texas Southwestern Medical Center, Dallas, TX, United States, ${ }^{2}$ Hamon \\ Center for Regenerative Science and Medicine, University of Texas Southwestern Medical Center, Dallas, TX, United States, \\ ${ }^{3}$ Spinal Cord and Brain Injury Research Group, Stark Neurosciences Research Institute, Indianapolis, IN, United States, \\ ${ }^{4}$ Department of Neurological Surgery, Indiana University School of Medicine, Indianapolis, IN, United States
}

OPEN ACCESS

Edited by:

Junfang Wu,

University of Maryland, Baltimore,

United States

Reviewed by:

Hedong Li,

Pennsylvania State University (PSU),

United States

Shuxin Li,

Temple University, United States

*Correspondence:

Chun-Li Zhang

chun-li.zhang@UTSouthwestern.edu

Specialty section:

This article was submitted to Cellular Neuropathology, a section of the journal Frontiers in Cellular

Neuroscience

Received: 14 February 2020 Accepted: 07 April 2020 Published: 24 April 2020

Citation:

Tai W, Xu X-M and Zhang C-L (2020) Regeneration Through in vivo Cell Fate Reprogramming for Neural Repair. Front. Cell. Neurosci. 14:107. doi: 10.3389/fncel.2020.00107
The adult mammalian central nervous system (CNS) has very limited regenerative capacity upon neural injuries or under degenerative conditions. In recent years, however, significant progress has been made on in vivo cell fate reprogramming for neural regeneration. Resident glial cells can be reprogrammed into neuronal progenitors and mature neurons in the CNS of adult mammals. In this review article, we briefly summarize the current knowledge on innate adult neurogenesis under pathological conditions and then focus on induced neurogenesis through cell fate reprogramming. We discuss how the reprogramming process can be regulated and raise critical issues requiring careful considerations to move the field forward. With emerging evidence, we envision that fate reprogramming-based regenerative medicine will have a great potential for treating neurological conditions such as brain injury, spinal cord injury (SCI), Alzheimer's disease (AD), Parkinson's disease (PD), and retinopathy.

Keywords: in vivo reprogramming, adult neurogenesis, traumatic brain injury (TBI), spinal cord injury (SCl), retinopathy, Alzheimer's diseases (AD), Parkinson's disease (PD)

\section{INTRODUCTION}

A functional central nervous system (CNS) consists of both neurons and glial cells. While neurons are responsible for generating and communicating electrical and chemical signals essential for neural networks, their activities are supported and modulated by surrounding glial cells (Rasband, 2016). Both neurons and glia can be affected by pathological conditions such as neural injuries and degenerative diseases. Disruption of functional neural networks is frequently permanent and is the underlying mechanism for many pathological symptoms for which no effective therapeutics exists. An unmet challenge is how to promote neural regeneration for CNS repair after various pathological conditions.

New neurons can be generated in the adult mammalian brain through a process of neurogenesis, which is positively or negatively regulated by pathological conditions (Zhao et al., 2008). However, innate neurogenesis is temporally and spatially restricted and is generally not sufficient for functional neural repair.

In vivo neural reprogramming is emerging as a promising new strategy for regenerative medicine (Chen et al., 2015; Smith and Zhang, 2015; Li and Chen, 2016; Smith et al., 2017; Torper and Götz, 2017; Barker et al., 2018; Wang and Zhang, 2018). This strategy employs genetic and epigenetic methods to reprogram resident glial cells into neuronal progenitors and 


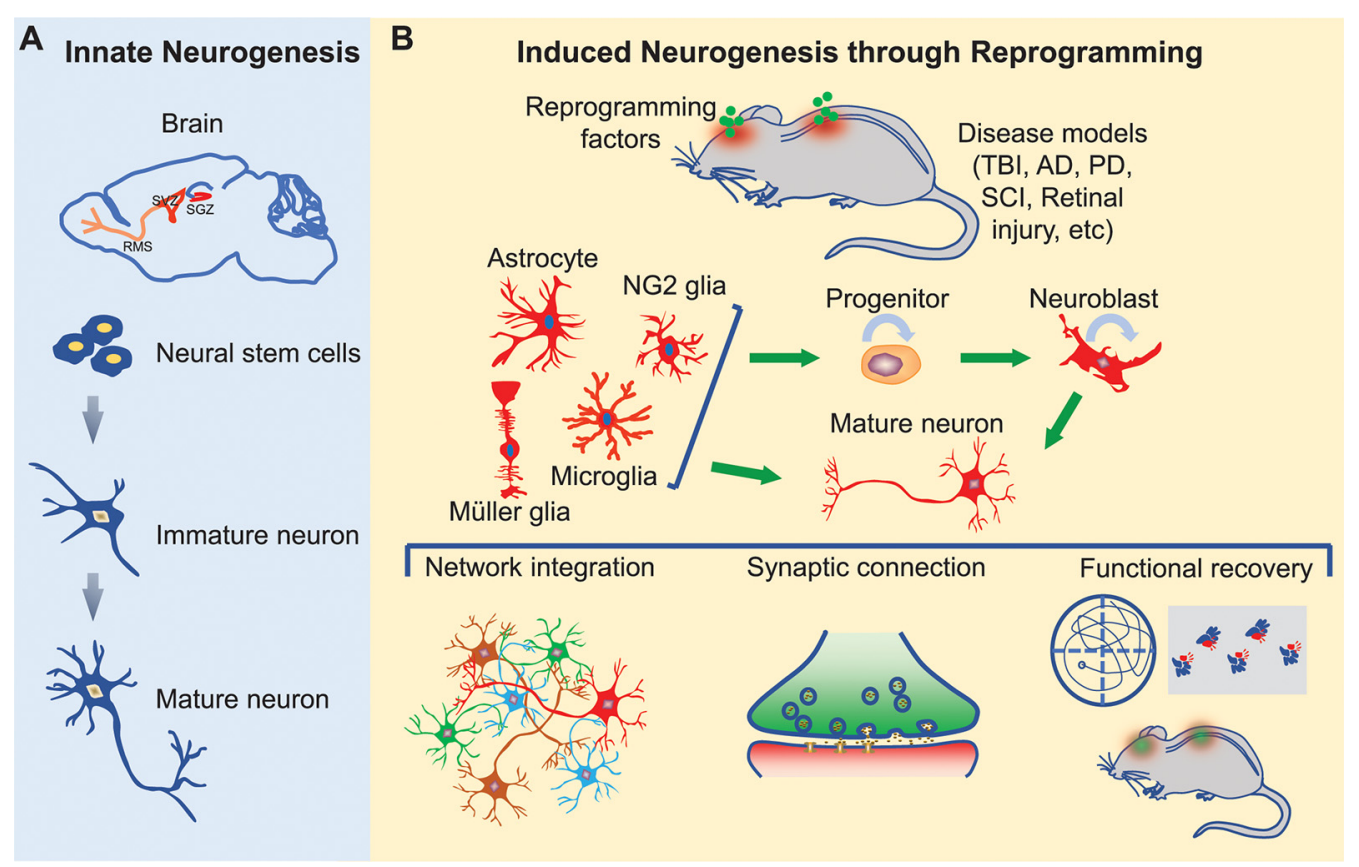

FIGURE 1 | Innate and induced neurogenesis in the adult central nervous system. (A) Innate adult neurogenesis mainly occurs in the subgranular zone (SGZ) of the hippocampal dentate gyrus and the subventricular zone (SVZ) of the lateral ventricle. Neural stem cells in these regions generate neurons under physiological and certain pathological conditions. Neurons generated in the SGZ remain in the dentate gyrus, whereas neurons originated from SVZ migrate along the rostral migratory stream (RMS) into the olfactory bulb. (B) Induced adult neurogenesis occurs through cell fate reprogramming in multiple regions of the brain, spinal cord, and retina. Resident glial cells can be directly reprogrammed into mature neurons or progenitors. The induced progenitors can expand through proliferation and eventually give rise to mature neurons. These induced neurons may integrate into the neural networks and promote functional recovery following neural injury or degeneration.

mature neurons in living animals. Unlike neurons that are frequently lost in response to pathological conditions, glial cells rather become activated and can form glial scars (Dixon, 2017; Gitler et al., 2017; Hayta and Elden, 2018). Though reactive glial cells may initially play beneficial roles, their persistent activation and scar formation are in general believed to hinder neural regeneration and may cause secondary damage to the surrounding tissues (Oyinbo, 2011; Freire, 2012; Anderson et al., 2016). In vivo fate reprogramming may turn reactive glial cells into useful neurons for damaged tissues.

Here, we will briefly review pathological regulations of innate neurogenesis and then focus on cell fate reprogramming for induced neurogenesis in the adult mammalian CNS (Figure 1).

\section{PATHOLOGICAL MODULATION OF THE INNATE NEUROGENESIS}

Decades of research showed that neurogenesis persists in restricted adult brain regions of many mammalian species: the subventricular zone (SVZ) of the lateral ventricle and the subgranular zone (SGZ) of the hippocampal dentate gyrus (DG; Zhao et al., 2008; Ziemka-Nałecz and Zalewska, 2012; Obernier et al., 2018). The adult neurogenesis process could be modulated by neural injuries and pathological conditions.

Traumatic brain injury (TBI) triggers rapid cell death predominantly in the cerebral cortex followed by secondary tissue loss in the hippocampus. TBI-induced changes in adult neurogenesis are well observed in rodents (Urrea et al., 2007; Wang X. et al., 2016). Such changes are further shown to be dependent on TBI severity (Wang X. et al., 2016). Both mild and moderate TBI are not sufficient to induce new neurons through neurogenesis, even though moderate TBI can promote the proliferation of innate neural stem cells. Only severe TBI can lead to enhanced neurogenesis indicated by both neural stem cell proliferation and the formation of new neurons. Very interestingly, studies of 11 human brain specimens showed that TBI induced proliferation of cells expressing markers for neural stem cells in the perilesional cortex of human brains, although whether new neurons are generated remains to be determined (Zheng et al., 2013).

Disruption of adult brain neurogenesis may represent a critical feature in many neurological diseases. Epilepsy, for example, modulates many cellular steps of adult neurogenesis including stem cell proliferation and migration and integration of newborn neurons (Jessberger and Parent, 2015). Adult neurogenesis was observed to be enhanced in the hippocampus of human patients with Alzheimer's disease (AD) and a mouse AD model (Jin et al., 2004a,b), whereas other mouse AD models rather showed decreased hippocampal neurogenesis (Donovan et al., 2006; Zhang et al., 2007). Adult hippocampal neurogenesis is similarly dysregulated in human Parkinson's disease (PD) and mouse models of PD (Desplats et al., 2012; Winner et al., 2012; He and Nakayama, 2015). 
Unlike the brain in which the phenomenon of adult neurogenesis is well-established, the spinal cord lacks any intrinsic ability to generate new neurons in adulthood (Horner et al., 2000). Spinal cord injury (SCI) can lead to the proliferation of multiple cell types including astrocytes and NG2 glia (also known as oligodendrocyte progenitor cells). However, none of these cells has been convincingly shown to generate mature neurons in vivo (Yamamoto et al., 2001; Horky et al., 2006). SCI also induces the proliferation of ependymal cells lining the central canal. When isolated and cultured in vitro, these cells exhibit stem cell-like properties that can form neurospheres and give rise to neurons, astrocytes, and oligodendrocytes (Meletis et al., 2008). Nevertheless, only astrocytes and oligodendrocytes are produced from injury-activated ependymal cells under in vivo conditions (Barnabé-Heider et al., 2010). It should be noted that, however, such a stem cell-like role of ependymal cells was recently questioned. The data from using more stringent genetic lineage tracing mouse lines rather showed that ependymal cells in either the adult brain or spinal cord lack any properties of stem cells and rarely contribute new cells to the injury site (Ren et al., 2017; Muthusamy et al., 2018; Shah et al., 2018).

\section{INDUCED NEUROGENESIS THROUGH FATE REPROGRAMMING IN VIVO}

Because of the restricted neurogenesis in the adult brain and a lack of any neurogenesis in the adult spinal cord, new strategies are devised to promote neural regeneration under neuropathological conditions. One emerging regenerative strategy is to reprogram the cell fate in vivo for the generation of new neurons. Such in vivo reprogramming is largely driven by ectopic expression of fate-determining transcriptional regulators. Functionally mature new neurons can be generated through this approach in several regions of the adult CNS (Table 1).

\section{Induced Neurons After Brain Injury}

Through in vivo screens of eight transcription factors (ASCL1, BRN2, KLF4, MYC, MYT1L, OCT4, SOX2, and ZFP521) and four microRNAs ( $m i R 9, m i R 124, m i R 125$, and miR128), the stem cell factor SOX2 was identified to be sufficient to reprogram resident astrocytes into functional neurons in the adult mouse striatum (Niu et al., 2013). These induced neurons fire action potentials and make synaptic connections with other local neurons. Subsequent studies revealed that SOX2mediated in vivo reprogramming passes through an expandable neural progenitor stage that is capable of generating multiple neurons from a single reprogrammed glia (Niu et al., 2015). Mechanistically, SOX2 induces the expression of transcription factors Ascl1 and Tlx, each of which is essential to the reprogramming process (Islam et al., 2015; Niu et al., 2015). In addition to astrocytes, Sox 2 alone is also sufficient to convert cortical NG2 glia into neurons especially following stab wound injury (Heinrich et al., 2014).

Glial cells can also be directly converted into neurons without passing through an expandable progenitor stage in the adult brain after injury. Such reprogramming is achieved by ectopic expression of transcription factors that are shown to play key roles during normal neurogenesis. Retrovirus-mediated expression of Neurod1 can efficiently convert cortical astrocytes or NG2 glia into functional neurons after brain injury (Guo et al., 2014). Very interestingly, cortical astrocytes were mainly converted into glutamatergic neurons, while NG2 glia were reprogrammed into both glutamatergic and GABAergic neurons. On the other hand, striatal microglia was recently shown to be reprogrammed by Neurod1-expressing lentivirus into DARPP32+ medium spiny neurons (Matsuda et al., 2019). Together, these results indicate that cellular context has a great influence on the identity of the induced neurons.

Recent data further showed that the efficiency of Neurod1mediated in vivo reprogramming of astrocytes could be significantly enhanced when Neurod1 was delivered through an AAV system (Zhang et al., 2018; Chen et al., 2020). Both endothelin1-induced ischemia cortical stroke and stab woundinduced cortical injury were shown to be nearly completely repaired. Remarkably, the regenerated neurons exhibited cortical layer-specific identities, formed functional neural circuits and rescued motor and memory deficits. Neurod1-mediated efficient neuronal conversion of astrocytes also leads to the regeneration of beneficial astrocytes, restoration of blood-brain-barriers, and reduction of neuroinflammation. Such broad and remarkable effects of Neurod1-dependent reprogramming of resident astrocytes, if confirmed, will revolutionize the therapeutic strategies for brain injuries.

Other neurogenic factors including Neurog2 and Ascl1 are also individually capable of inducing new neurons in the adult mouse brain (Grande et al., 2013; Liu et al., 2015; Gascon et al., 2016). The Neurog2-mediated in vivo reprogramming could be significantly enhanced by local exposure to growth factors or by co-expression of $\mathrm{Bcl}-2$ and anti-oxidative treatments (Grande et al., 2013; Gascon et al., 2016). The latter treatments were revealed to act through inhibition of the lipid peroxidation-mediated ferroptosis pathway (Gascon et al., 2016). In addition to single factormediated fate reprogramming, the combination of Ascl1 with Brn2 and Myt1l through AAV-mediated expression also led to the conversion of striatal astrocytes or NG2 glia to neurons that could functionally integrate into the local circuits (Torper et al., 2013; Pereira et al., 2017). Upon stab wound injury, AAV-mediated expression of Neurog2 and Nurr1 in a Cre-dependent manner can robustly convert cortical astrocytes into functional pyramidal neurons (Mattugini et al., 2019). Remarkably, these induced pyramidal neurons exhibit cortical layer-specific identity indicated by lamina-specific marker expression and long-distance axonal projections. It will be extremely interesting to investigate whether these new cortical neurons can lead to functional improvements after TBI.

After TBI and retrovirus-mediated ectopic expression of the reprogramming OSKM factors (Oct4, Sox2, Klf4, and $c M y c$ ), cells resembling induced pluripotent stem cells (iPSCs) could be induced from reactive glial cells in the injured cortex (Gao et al., 2016). These cells extensively proliferated and spontaneously differentiated into neural stem cells and neurons. Such extensive 


\begin{tabular}{|c|c|c|c|c|}
\hline Region/Disease model & Cell source & Reprogramming factors & $\begin{array}{l}\text { Functional properties of the } \\
\text { induced neurons }\end{array}$ & References \\
\hline Cortex/Stab injury; AD & Glia & Neurod1 & $\begin{array}{l}\text { Functional synapses with surrounding } \\
\text { neurons }\end{array}$ & Guo et al. (2014) \\
\hline Cortex/Stab injury & NG2 glia & Sox2 & $\begin{array}{l}\text { Synaptic inputs from innate neurons } \\
\text { near the injury site }\end{array}$ & Heinrich et al. (2014) \\
\hline Cortex/Stab injury & Glia & Neurog2; Bcl-2; $\alpha$-Tocotrienol & Complex morphology & Gascon et al. (2016) \\
\hline Cortex/Controlled cortical impact injury & Glia & Oct4; KIf4; Sox2;c-Myc & $\begin{array}{l}\text { Electrophysiology; network-integration; } \\
\text { reduction of tissue cavity }\end{array}$ & Gao et al. (2016) \\
\hline Cortex/Stab injury & Astrocytes & Neurod1 & $\begin{array}{l}\text { Brain repair with a reduction of tissue } \\
\text { loss }\end{array}$ & Zhang et al. (2018) \\
\hline Cortex/Stab Injury & Astrocytes & Neurog2; Nurr1 & $\begin{array}{l}\text { Cortical layer-specific identity; synaptic } \\
\text { connections; long axonal projections }\end{array}$ & Mattugini et al. (2019) \\
\hline Cortex/Ischemic injury & Astrocytes & Neurod1 & $\begin{array}{l}\text { Synaptic connections; long-range } \\
\text { axonal projections; improvement of } \\
\text { motor and cognitive functions }\end{array}$ & Chen et al. (2020) \\
\hline Striatum/Injection injury & Astrocytes & sox2 & Electrophysiology; network integration & Niu et al. (2013) \\
\hline Striatum/6-OHDA lesion & $\begin{array}{l}\text { Human embryonic } \\
\text { fibroblasts/human fetal lung fibroblast }\end{array}$ & Ascl1; Brn2a; Myt1l & Innervation into host tissues & Torper et al. (2013) \\
\hline Striatum/Injection injury & Astrocytes & sox2 & $\begin{array}{l}\text { Network-integration with inputs from } \\
\text { presynaptic neurons }\end{array}$ & Niu et al. (2015) \\
\hline Striatum/Injection injury & NG2 glia & Ascl1; Lmx1a; Nurr1 & $\begin{array}{l}\text { Innervation from pre-existing local } \\
\text { circuitry }\end{array}$ & Torper et al. (2015) \\
\hline Striatum; midbrain/6-OHDA & NG2 glia & Ascl1; Lmx1a; Nurr1; etc. & Electrophysiology; network integration & Pereira et al. (2017) \\
\hline Striatum/PD & Astrocytes & $\begin{array}{l}\text { Neurod1; Ascl1; Lmx1a; } \\
\text { miR218 }\end{array}$ & $\begin{array}{l}\text { Electrophysiology; rescue of } \\
\text { spontaneous motor behavior }\end{array}$ & Rivetti di Val Cervo et al. (2017) \\
\hline Striatum/PD & Astrocytes & Ascl1; Pitx3; Lmx1a; Nurr1 & $\begin{array}{l}\text { Electrophysiology; rescue of } \\
\text { Parkinsonian phenotypes }\end{array}$ & Yoo et al. (2017) \\
\hline Striatum/Injection injury & Striatal neurons & $\begin{array}{l}\text { SOX2; NURR1; FOXA2; } \\
\text { LMX1A; Valproic acid }\end{array}$ & Electrophysiology; network integration & Niu et al. (2018) \\
\hline Striatum/Injection injury & Microglia & Neurod1 & $\begin{array}{l}\text { Integration into brain circuits with } \\
\text { synaptic connections. }\end{array}$ & Matsuda et al. (2019) \\
\hline Striatum; Cortex/Stab injury; ischemia & Non-neuronal cells & Neurog2; growth factors & Region-specific differentiation & Grande et al. (2013) \\
\hline Dorsal midbrain; Striatum; Cortex/Stab or injection injury & Astrocytes & Ascl1 & Electrophysiology; network integration & Liu et al. (2015) \\
\hline Dentate gyrus/AD & Astrocytes & microRNA-302/367 & $\begin{array}{l}\text { Electrophysiology; improvement of } \\
\text { spatial learning and memory }\end{array}$ & Ghasemi-Kasman et al. (2018) \\
\hline Spinal cord/SCI & Astrocytes & sox2 & Synapse-forming interneurons & Su et al. (2014) \\
\hline Spinal cord/SCl & Astrocytes & SOX2 & $\begin{array}{l}\text { Synaptic connections with local } \\
\text { neurons }\end{array}$ & Wang L. L. et al. (2016) \\
\hline Retina/Retinal injury & Müller glia & Ascl1 & Amacrine, bipolar, or photoreceptors & Ueki et al. (2015) \\
\hline Retina/Retinal injury & Müller glia & Ascl1; Trichostatin-A & $\begin{array}{l}\text { Inner retinal neurons; synapses with } \\
\text { host retinal neurons; response to light }\end{array}$ & Jorstad et al. (2017) \\
\hline Retina/Congenital blindness & Müller glia & $\beta$-catenin; $\mathrm{Otx} 2 ; \mathrm{Crx} ; \mathrm{Nrl}$ & $\begin{array}{l}\text { Rod photoreceptors; restoration of } \\
\text { visual responses }\end{array}$ & Yao et al. (2018) \\
\hline
\end{tabular}

TABLE 1 | Induced neurogenesis in the adult central nervous system. 
proliferation and neural differentiation of iPSCs may be needed to fill in the cavity caused by TBI; however, tumorigenesis and simultaneous generation of non-neural cells in the brain need to be prevented for this approach to be useful for brain repair.

\section{Induced Neurons for Alzheimer's Disease}

Because of the progressive and pleiotropic neurodegeneration observed in $\mathrm{AD}$, local induction of new neurons as a therapeutic approach may be challenging for this type of neurologic disease. Nonetheless, in vivo reprogramming of reactive glial cells into neurons were attempted in mouse models of AD. Similar to acute brain injuries, $\mathrm{AD}$ and other neurodegenerative diseases are frequently accompanied by reactive astrocytosis. The reactive astrocytes were capable of being reprogrammed into neurons by ectopic expression of Neurod 1 in the cortex of $5 x F A D$ mice (Guo et al., 2014). Electrophysiological recordings of induced neurons in $5 \mathrm{xFAD}$ brain slices showed that the induced neurons were functional and made synaptic connections with surrounding neurons. Interestingly, the number of Neurod1-induced neurons was higher in aged and diseased brains than in young and healthy ones, suggesting that more reactive glial cells observed in aged and diseased brains might provide more cells for reprogramming in vivo (Guo et al., 2014). In another AD mouse model that was induced by intraventricular injection of streptozocin, $m i R-302 / 367$ ectopic expression in combination with valproate treatment was reported to induce neurons from reactive astrocytes in the dentate gyrus (Ghasemi-Kasman et al., 2018). Such treatments also led to improved performance on a behavioral task measuring spatial learning and memory, implicating a new therapeutic strategy for $\mathrm{AD}$ patients.

\section{Induced Neurons for Parkinson's Disease}

The cellular basis for PD is the loss of dopaminergic neurons in the midbrain. Transplantation of this type of neurons ameliorated many pathological symptoms associated with PD. This raised the possibility that new dopaminergic neurons derived from fate reprogramming in vivo may be a therapeutic alternative to cell transplantation. Several studies explored the feasibility of inducing new dopaminergic neurons in the adult mouse brains. A combination of Ascl1, Lmx1a, and Nurr1 (ALN) was shown to be sufficient to reprogram mouse fibroblasts and astrocytes into dopaminergic neurons in culture (Addis et al., 2011; Caiazzo et al., 2011). When this combination was introduced into the adult mouse striatum, however, it completely failed to induce any dopaminergic neurons from either astrocytes or NG2 glia (Torper et al., 2015). Subsequent studies further revealed that ALN or many other combinations of fate-determining factors also failed to induce dopaminergic neurons, although they could very robustly convert resident NG2 glia into interneurons in the striatum (Pereira et al., 2017).

In contrast, in a mouse PD model that was induced by 6-hydroxydopamine (6-OHDA), striatal astrocytes were reported to be directly converted into dopaminergic neurons when a cocktail of four transcription factors (Neurod1, Ascl1, Lmx1a, and miR218) was delivered into the ipsilateral striatum (Rivetti di Val Cervo et al., 2017). The in vivo induced dopaminergic neurons were excitable and ameliorated some aspects of motor deficits such as gait impairments. Using a different set of transcription factors (Ascl1, Pitx3, Lmx1a, and Nurr1) and in combination with electromagnetic gold nanoparticles (AuNPs) and the presence of specific electromagnetic fields, Yoo et al. (2017) reported that dopaminergic neurons could be converted from resident striatal astrocytes in MPTP- or 6-OHDA-induced PD mouse models. Importantly, such combinatorial treatments also alleviated locomotor deficits that were observed in these mouse PD models. Results of the above two reports showed the therapeutic potential of in vivo reprogrammed dopaminergic neurons for PD.

Taking a slightly different strategy, Niu et al. (2018) reported that functional dopaminergic neurons could also be generated in the adult mouse striatum. Unexpectedly, systematic lineage mappings in several transgenic mouse lines rather discovered that these induced dopaminergic neurons were originated from resident striatal neurons but not from any glial cells. Such phenotypic reprogramming revealed unprecedented plasticity of mature neurons and implicated an alternative strategy to rewire brain circuits in the adult. It will be interesting to determine the biological effect of such reprogrammed dopaminergic neurons in PD.

\section{Induced Neurons After Spinal Cord Injury}

Spinal cord injuries (SCI) lead to the disruption of neural circuits and the loss of propriospinal neurons. The addition of new neurons may well serve as relays that could form neural circuits for functional improvements. Ectopic expression of SOX2 induced DCX+ neuroblasts in the adult spinal cord after injury (Su et al., 2014). Lineage tracing confirmed an astrocyte origin for the induced neuroblasts. These neuroblasts could expand through proliferation and eventually become mature neurons that formed connections with spinal motor neurons. Through in vivo screens of additional 17 factors, the p53-p21 signaling pathway was identified to be critical for controlling the expansion of SOX2-induced neuroblasts in the adult spinal cord (Wang L. L. et al., 2016). Downregulation of either p53 or p21 led to a greatly increased number of $S O X 2$-induced neuroblasts and mature neurons. The ability to expand the number of induced neurons may be therapeutically advantageous since a SCI frequently leads to the death of many propriospinal neurons. Interestingly, a majority of these SOX2-induced neurons are VGLUT2+ excitatory interneurons (Wang L. L. et al., 2016), which are in sharp contrast to those in the striatum that are mainly GABAergic interneurons (Niu et al., 2015). VGLUT2+ interneurons are essential components of the locomotor circuitry and play an important role in the proper organization of the spinal locomotor network (Borgius et al., 2014). As such, it is conceivable that the induced VGLUT2 excitatory interneurons may well be capable of forming relay circuits (Courtine et al., 2008; Abematsu et al., 2010) with ascending and descending pathways that are frequently disrupted by SCI.

\section{Induced Neurons for Retinopathy}

Müller glial cells (MGs) are the major glial cells in the retina, an integral part of the mammalian CNS that lacks any neurogenesis 
under both physiological and pathological conditions. Ectopic Ascl1 in young transgenic mice renders MGs to generate retinal neurons including amacrine and bipolar cells and photoreceptors after injury (Ueki et al., 2015). However, such Ascl1-mediated reprogramming of MGs is restricted to the first 2 weeks after birth. An epigenetic barrier may account for the reprogramming failure of adult MGs since the chromatin is less accessible in these cells than those young MGs (Ueki et al., 2015). Indeed, when treated with a histone deacetylase inhibitor that leads to increased histone acetylation and an open chromatin structure, ectopic Ascl1 can reprogram MGs to generate retinal neurons after retinal injury in the adult mice (Jorstad et al., 2017). Importantly, these induced neurons can make synapses with resident neurons and respond to light stimulation, indicating functional integration into the neural network. It is not clear, though, why the induce neurons are mainly bipolar cells, since lineage tracing shows that Ascl1-expressing progenitors can give rise to multiple neuronal subtypes during retinogenesis (Brzezinski et al., 2011).

Taking a two-step approach, Yao et al. (2018) show that activated MGs can be directly reprogrammed into functional rod photoreceptors in the adult mice. AAV-mediated ectopic expression of $\beta$-catenin enables the adult MGs to proliferate without prior injury (Yao et al., 2016). However, a majority of these activated MGs undergo cell death and rarely generate any new retinal neurons. Remarkably, subsequent AAV-mediated expression of $\mathrm{Otx} 2, \mathrm{Cr} x$, and $\mathrm{Nrl}$ in these activated MGs allows them to produce rod photoreceptors, which are capable of restoring visual responses in a mouse model of congenital blindness (Yao et al., 2018). It will be certainly interesting to examine whether other retinal cell types, such as retinal ganglion cells, can be similarly reprogrammed from MGs by using a different set of fate-determining factors.

\section{THE INFLUENCE OF CELLULAR CONTEXT ON FATE REPROGRAMMING}

Emerging evidence indicates that cellular contexts, such as senescence, mitochondria dynamics, and autophagy, play critical roles during the reprogramming process.

\section{Senescence}

Senescence is characterized by permanent cell cycle arrest. Cells in senescence secrete inflammatory cytokines, chemokines, and growth factors that are together referred to as the senescenceassociated secretory phenotype (SASP; Childs et al., 2015). Senescence plays critical roles in tissue repair, embryonic development, as well as aging-related diseases (Loeser, 2009; Storer et al., 2013; Demaria et al., 2014). In a transgenic mouse model expressing the reprogramming OSKM factors for iPSCs, the extent of cell senescence is found to be positively correlated with the efficiency of cell reprogramming in vivo (Mosteiro et al., 2016). OSKM-induced senescent cells secrete interleukin6 , which creates a favorable microenvironment that facilitates reprogramming in adult mice. Mechanistic studies further reveal that Ink4a is critical for the induction of cell senescence and reprogramming; however, it is dispensable when p53 is removed
(Mosteiro et al., 2018). Consistent with a positive role of cell senescence, the frequency of OSKM-induced reprogramming is increased in aged mice (Mosteiro et al., 2016).

\section{Mitochondrial Dynamics}

Mitochondrion plays critical roles in many cellular processes, such as metabolism, energy production, generation of reactive oxygen species (ROS), cell signaling, and apoptosis (Sarsour et al., 2009; Osellame et al., 2012). It is also emerging as a key player in cell fate determination, maintenance of pluripotency, and cell reprogramming (Folmes et al., 2012; Maryanovich and Gross, 2013; Prieto and Torres, 2017). Mitochondrial respiratory dysfunction triggered by mutant mtDNAs blocks cellular reprogramming, though it does not affect the maintenance of the pluripotent state (Yokota et al., 2015). Direct reprogramming of somatic cells to neurons induces a metabolic switch that leads to high levels of oxidative stress and ferroptosis-dependent cell death. As such, reduction of ROS through Bcl-2 ectopic expression or treatments with antioxidants potently promote neuronal reprogramming both in culture and in a mouse model of TBI (Gascon et al., 2016). On the other hand, mitochondrial fission accompanies the early phase of cell reprogramming (Prieto et al., 2016). Blocking the pro-fission factor Drp1 hinders the production of iPSCs. It should be interesting to examine whether mitochondria dynamics are associated with neural reprogramming in the adult CNS.

\section{Autophagy}

Autophagy is a cellular process that degrades cytoplasmic components and organelles by the lysosome to maintain cellular homeostasis (Mizushima and Levine, 2010). Recent in vitro and in vivo studies reveal critical roles of autophagy in the regulation of adult neurogenesis and reprogramming (Tang, 2013; Wang et al., 2015). Conditional deletion of Fip200, an essential gene for the induction of mammalian autophagy, leads to impaired autophagy, increased mitochondrial number, and elevated ROS in postnatal mouse neural stem cells. Consequently, stem cell maintenance and neuronal differentiation are severely impaired in these mice; and, such impairments can be rescued by reducing ROS levels through treatments with an antioxidant (Wang et al., 2013). Beclin1, a gene required for autophagosome formation, is similarly required for adult neurogenesis in the lateral ventricle (Yazdankhah et al., 2014). On the other hand, iPSC reprogramming requires mitochondrial clearance mediated by the AMPK-dependent but Atg5-independent autophagic process (Ma et al., 2015). It remains to be determined what roles of autophagy play during neural reprogramming in vivo.

\section{ISSUES TO CONSIDER}

Despite the recent major progresses, several key issues require considerations to move forward the field of neural reprogramming in vivo.

\section{Cell Origin for Reprogrammed Neurons}

The cell origin for the induced neurons requires thorough investigation and confirmation. Unlike in vitro reprogramming 
that can start from a relatively pure population of cells and the fate-switch can be directly observed under a microscope, in vivo reprogramming occurs in a very complex microenvironment consisting of multiple cell types including resident neurons. It is essential to use well-established lineage tracing methods or time-lapse imaging to follow the reprogramming process and confirm the cell origin for the induced neurons (Niu et al., 2013, 2018; Heinrich et al., 2014; Pilz et al., 2018). If the induced neurons originate from reactive and proliferative glial cells, the BrdU- or EdU-based labeling method is a simple and validated way to confirm that the induced neurons are indeed newly generated (Grande et al., 2013; Niu et al., 2013; Heinrich et al., 2014; Wang L. L. et al., 2016; Mattugini et al., 2019). Based on our years' experiences working with lentiviruses and AAVs, it is not reliable to use the virus-based reporters as a sole tracing method for in vivo induced neurons. Despite using cell-typespecific promoters (such as the human GFAP promoter), the attached sequences (such as GFP vs. reprogramming factors) can significantly affect cell specificity, consistent with a previous report in transgenic mice (Su et al., 2004). Retroviruses, on the other hand, can induce fusion of virus-transduced microglia with resident mature neurons (Ackman et al., 2006). As such, experiments should be conducted to exclude the possibility that resident neurons are mistakenly considered as induced neurons.

\section{Molecular and Cellular Mechanisms Underlying Neural Reprogramming in vivo}

Emerging evidence indicates that multiple pathways regulate the in vivo reprogramming process. The neurogenic factor Ascl1 and the nuclear receptor $T l x$ are found to be essential mediators of SOX2-dependent reprogramming of astrocytes in the adult brain (Niu et al., 2015). Through a series of in vivo screens, the p53-p21 pathway was identified to function as a critical checkpoint for glial reprogramming in the adult mouse spinal cord (Wang L. L. et al., 2016). Neurog2-mediated direct neuronal reprogramming can be remarkably promoted by a cocktail of growth factors or coexpression of $\mathrm{Bcl}-2$ and anti-oxidative treatments in the adult brain (Grande et al., 2013; Gascon et al., 2016). Reprogramming of microglia by Neurod 1 is accompanied by changes in the epigenetic landscape, which gradually leads to loss of microglial traits and acquisition of neuronal identity (Matsuda et al., 2019). Inhibition of histone deacetylases, which promotes chromatin accessibility, is required for Ascll to convert adult MGs into neurons in the injured retina (Jorstad et al., 2017). With recent advancements in Omics tools, such as scRNA-seq, ChIP-seq, and ATAC-seq, more molecular details on the reprogramming process are expected to emerge shortly. Cautions should be taken, however, when extrapolating results from cell culture models, since many of them might not be reproducible under in vivo conditions. For example, both mouse fibroblasts and astrocytes can be efficiently reprogrammed into dopaminergic neurons in culture (Addis et al., 2011; Caiazzo et al., 2011). These same reprogramming factors, nonetheless, completely failed to induce any dopaminergic neurons from glial cells in the adult mouse brain (Torper et al., 2015; Pereira et al., 2017).
Intriguingly, various glial cells can be efficiently reprogrammed by diverse factors into brain region-specific neuronal subtypes. For example, a combination of Neurog2 and Nurr1 reprograms adult astrocytes into diverse neuronal subtypes with cortical layer-specificity and precise long-distance axonal projections (Mattugini et al., 2019). Such feats can be similarly accomplished by ectopic expression of Neurod 1 in the adult cortex with or without prior injury (Chen et al., 2020; Liu et al., 2020). In the adult striatum, Neurod1 can efficiently reprogram both astrocytes and microglia into mature striatal neurons (Matsuda et al., 2019; Liu et al., 2020). These striatal neurons can also be converted from oligodendrocytes by microRNA-mediated knockdown of Ptbp1 (Weinberg et al., 2017). How do these different reprogramming factors generate similar or identical subtypes of neurons from reactive or non-reactive astrocytes, microglia, and oligodendrocytes? How is region-specificity established for the glia-converted neurons? Do these diverse glial cells retain brain region-specificity that potentially mediates such phenomenal fate-switch when a neurogenic factor is ectopically expressed? If so, how could they still maintain region- and subtype-specificity after brain injury? How could microglia, which is not even derived from the neural lineage, be efficiently reprogrammed into neuronal subtypes as those from astrocytes by the same reprogramming factor such as Neurod1? Since most, if not all, of the developmental cues for axon guidance, are diminished in the adult CNS, how do these newly reprogrammed neurons make precise axonal projections especially after neural injuries? Answers to these questions will provide critical insights into the cellular mechanisms for neural reprogramming in vivo.

\section{Biological Functions of Neural Reprogramming}

In vivo neural reprogramming has shown promising biological functions for stroke-induced brain injury (Chen et al., 2020), chemical-induced PD (Rivetti di Val Cervo et al., 2017; Yoo et al., 2017), and a genetic mouse model of blindness (Yao et al., 2018). If confirmed, these advancements will bring unprecedented regeneration-based therapies for many other neural injuries and neurological diseases. Nonetheless, these new neurons may not lead to the recovery of lost memories, although they may help form new ones. It remains unclear what role of newly reprogrammed neurons play during functional recovery since the reprogramming of glial cells not only produces new neurons but also alters the microenvironment. After an injury or under degenerative conditions, reactive gliosis play critical roles in modulating tissue damage and neural regeneration (Okada et al., 2006; Sofroniew, 2009; Robel et al., 2011; Karimi-Abdolrezaee and Billakanti, 2012). Of note, scar formation and secretion of chondroitin sulfate proteoglycans (CSPG) by reactive glial cells are inhibitory for functional improvement post neural injury. Attenuation of reactive gliosis or reducing CSPG activity improves post-traumatic regeneration (Wilhelmsson et al., 2004; Lang et al., 2015), whereas increasing reactive gliosis worsens brain injuries (Mori et al., 2008). As such, it will be interesting to tease out the respective contributions of new neurons and 
the environmental changes to functional recovery post injuries or degenerations. On the other hand, it also remains to be determined whether glia-converted neurons play any detrimental effect, since these new neurons may well lead to disruption of preexisting neural circuits or formation of abnormal ones. Rehabilitation may be needed for the new neurons to incorporate into functional neural circuits.

\section{CONCLUDING REMARKS}

In vivo neural reprogramming has achieved impressive progress, ranging from generation of diverse glia-converted neurons in multiple CNS regions to functional improvements for certain neurological conditions. Such a reprogramming-based approach may kill two birds with one stone: regeneration of functional neurons and modulation of pathological microenvironment. Nonetheless, efforts should be taken to vigorously validate the cell origin for the claimed new neurons and to tease out the molecular and cellular mechanisms underlying the reprogramming progress. The results of these efforts will lay a solid scientific foundation to move in vivo cell fate reprogramming towards neural repair.

\section{REFERENCES}

Abematsu, M., Tsujimura, K., Yamano, M., Saito, M., Kohno, K., Kohyama, J., et al. (2010). Neurons derived from transplanted neural stem cells restore disrupted neuronal circuitry in a mouse model of spinal cord injury. J. Clin. Invest. 120, 3255-3266. doi: 10.1172/jci42957

Ackman, J. B., Siddiqi, F., Walikonis, R. S., and LoTurco, J. J. (2006). Fusion of microglia with pyramidal neurons after retroviral infection. J. Neurosci. 26, 11413-11422. doi: 10.1523/JNEUROSCI.3340-06.2006

Addis, R. C., Hsu, F. C., Wright, R. L., Dichter, M. A., Coulter, D. A., and Gearhart, J. D. (2011). Efficient conversion of astrocytes to functional midbrain dopaminergic neurons using a single polycistronic vector. PLoS One 6:e28719. doi: 10.1371/journal.pone.0028719

Anderson, M. A., Burda, J. E., Ren, Y., Ao, Y., O’Shea, T. M., Kawaguchi, R., et al. (2016). Astrocyte scar formation aids central nervous system axon regeneration. Nature 532, 195-200. doi: 10.1038/nature17623

Barker, R. A., Götz, M., and Parmar, M. (2018). New approaches for brain repair-from rescue to reprogramming. Nature 557, 329-334. doi: 10.1038/s41586-018-0087-1

Barnabé-Heider, F., Göritz, C., Sabelström, H., Takebayashi, H., Pfrieger, F. W., Meletis, K., et al. (2010). Origin of new glial cells in intact and injured adult spinal cord. Cell Stem Cell 7, 470-482. doi: 10.1016/j.stem.2010.07.014

Borgius, L., Nishimaru, H., Caldeira, V., Kunugise, Y., Low, P., Reig, R., et al. (2014). Spinal glutamatergic neurons defined by EphA4 signaling are essential components of normal locomotor circuits. J. Neurosci. 34, 3841-3853. doi: 10.1523/JNEUROSCI.4992-13.2014

Brzezinski, J. A. T., Kim, E. J., Johnson, J. E., and Reh, T. A. (2011). Ascll expression defines a subpopulation of lineage-restricted progenitors in the mammalian retina. Development 138, 3519-3531. doi: 10.1242/dev.064006

Caiazzo, M., Dell'Anno, M. T., Dvoretskova, E., Lazarevic, D., Taverna, S., Leo, D., et al. (2011). Direct generation of functional dopaminergic neurons from mouse and human fibroblasts. Nature 476, 224-227. doi: 10.1038/nature10284

Chen, Y. C., Ma, N. X., Pei, Z. F., Wu, Z., Do-Monte, F. H., Keefe, S., et al. (2020). A NeuroD1 AAV-based gene therapy for functional brain repair after ischemic injury through in vivo astrocyte-to-neuron conversion. Mol. Ther. 28, 217-234. doi: 10.1016/j.ymthe.2019.09.003

Chen, G., Wernig, M., Berninger, B., Nakafuku, M., Parmar, M., and Zhang, C. L. (2015). In vivo reprogramming for brain and spinal cord repair. eNeuro 2:ENEURO.0106-15.2015. doi: 10.1523/eneuro.0106-15.2015

\section{AUTHOR CONTRIBUTIONS}

WT and C-LZ wrote the manuscript and X-MX commented on the manuscript.

\section{FUNDING}

The research in the Zhang laboratory is supported by The Welch Foundation (I-1724), the Decherd Foundation, the Pape Adams Foundation, Kent Waldrep Foundation Center for Basic Research on Nerve Growth and Regeneration, and NIH Grants (NS099073, NS092616, NS088095, and NS093502). Research in the $\mathrm{Xu}$ laboratory is supported by NIH 1R01 100531, 1R01 NS103481, and Merit Review Award I01 BX002356, I01 BX003705, I01 RX002687 from the U.S. Department of Veterans Affairs.

\section{ACKNOWLEDGMENTS}

We thank members of the Zhang laboratory for insightful discussions. C-LZ is a WW Caruth, Jr. Scholar in Biomedical Research.

Childs, B. G., Durik, M., Baker, D. J., and van Deursen, J. M. (2015). Cellular senescence in aging and age-related disease: from mechanisms to therapy. Nat. Med. 21, 1424-1435. doi: 10.1038/nm.4000

Courtine, G., Song, B., Roy, R. R., Zhong, H., Herrmann, J. E., Ao, Y., et al. (2008). Recovery of supraspinal control of stepping via indirect propriospinal relay connections after spinal cord injury. Nat. Med. 14, 69-74. doi: 10.1038/nm1682

Demaria, M., Ohtani, N., Youssef, S. A., Rodier, F., Toussaint, W., Mitchell, J. R., et al. (2014). An essential role for senescent cells in optimal wound healing through secretion of PDGF-AA. Dev. Cell 31, 722-733. doi: 10.1016/j.devcel. 2014.11.012

Desplats, P., Spencer, B., Crews, L., Pathel, P., Morvinski-Friedmann, D., Kosberg, K., et al. (2012). $\alpha$-Synuclein induces alterations in adult neurogenesis in Parkinson disease models via p53-mediated repression of Notch1. J. Biol. Chem. 287, 31691-31702. doi: 10.1074/jbc.m112.354522

Dixon, K. J. (2017). Pathophysiology of traumatic brain injury. Phys. Med. Rehabil. Clin. N. Am. 28, 215-225. doi: 10.1016/j.pmr.2016.12.001

Donovan, M. H., Yazdani, U., Norris, R. D., Games, D., German, D. C., and Eisch, A. J. (2006). Decreased adult hippocampal neurogenesis in the PDAPP mouse model of Alzheimer's disease. J. Comp. Neurol. 495, 70-83. doi: $10.1002 /$ cne. 20840

Folmes, C. D., Dzeja, P. P., Nelson, T. J., and Terzic, A. (2012). Metabolic plasticity in stem cell homeostasis and differentiation. Cell Stem Cell 11, 596-606. doi: 10.1016/j.stem.2012.10.002

Freire, M. A. (2012). Pathophysiology of neurodegeneration following traumatic brain injury. West Indian Med. J. 61, 751-755.

Gao, X., Wang, X., Xiong, W., and Chen, J. (2016). In vivo reprogramming reactive glia into iPSCs to produce new neurons in the cortex following traumatic brain injury. Sci. Rep. 6:22490. doi: 10.1038/srep22490

Gascon, S., Murenu, E., Masserdotti, G., Ortega, F., Russo, G. L., Petrik, D., et al. (2016). Identification and successful negotiation of a metabolic checkpoint in direct neuronal reprogramming. Cell Stem Cell 18, 396-409. doi: 10.1016/j. stem.2015.12.003

Ghasemi-Kasman, M., Shojaei, A., Gol, M., Moghadamnia, A. A., Baharvand, H., and Javan, M. (2018). miR-302/367-induced neurons reduce behavioral impairment in an experimental model of Alzheimer's disease. Mol. Cell. Neurosci. 86, 50-57. doi: 10.1016/j.mcn.2017.11.012

Gitler, A. D., Dhillon, P., and Shorter, J. (2017). Neurodegenerative disease: models, mechanisms, and a new hope. Dis. Model Mech. 10, 499-502. doi: 10.1242/dmm.030205 
Grande, A., Sumiyoshi, K., López-Juárez, A., Howard, J., Sakthivel, B., Aronow, B., et al. (2013). Environmental impact on direct neuronal reprogramming in vivo in the adult brain. Nat. Commun. 4:2373. doi: 10.1038/ncomms3373

Guo, Z., Zhang, L., Wu, Z., Chen, Y., Wang, F., and Chen, G. (2014). In vivo direct reprogramming of reactive glial cells into functional neurons after brain injury and in an Alzheimer's disease model. Cell Stem Cell 14, 188-202. doi: 10.1016/j. stem.2013.12.001

Hayta, E., and Elden, H. (2018). Acute spinal cord injury: a review of pathophysiology and potential of non-steroidal anti-inflammatory drugs for pharmacological intervention. J. Chem. Neuroanat. 87, 25-31. doi: 10.1016/j. jchemneu.2017.08.001

He, X. J., and Nakayama, H. (2015). Transiently impaired neurogenesis in MPTP mouse model of Parkinson's disease. Neurotoxicology 50, 46-55. doi: 10.1016/j. neuro.2015.07.007

Heinrich, C., Bergami, M., Gascon, S., Lepier, A., Viganò, F., Dimou, L., et al. (2014). Sox2-mediated conversion of NG2 glia into induced neurons in the injured adult cerebral cortex. Stem Cell Reports 3, 1000-1014. doi: 10.1016/j. stemcr.2014.10.007

Horky, L. L., Galimi, F., Gage, F. H., and Horner, P. J. (2006). Fate of endogenous stem/progenitor cells following spinal cord injury. J. Comp. Neurol. 498, 525-538. doi: 10.1002/cne.21065

Horner, P. J., Power, A. E., Kempermann, G., Kuhn, H. G., Palmer, T. D., Winkler, J., et al. (2000). Proliferation and differentiation of progenitor cells throughout the intact adult rat spinal cord. J. Neurosci. 20, 2218-2228. doi: 10.1523/JNEUROSCI.20-06-02218.2000

Islam, M. M., Smith, D. K., Niu, W., Fang, S., Iqbal, N., Sun, G., et al. (2015). Enhancer analysis unveils genetic interactions between TLX and SOX2 in neural stem cells and in vivo reprogramming. Stem Cell Reports 5, 805-815. doi: 10.1016/j.stemcr.2015.09.015

Jessberger, S., and Parent, J. M. (2015). Epilepsy and adult neurogenesis. Cold Spring Harb. Perspect. Biol. 7:a020677. doi: 10.1101/cshperspect.a020677

Jin, K., Galvan, V., Xie, L., Mao, X. O., Gorostiza, O. F., Bredesen, D. E., et al. (2004a). Enhanced neurogenesis in Alzheimer's disease transgenic (PDGF-APPSw,Ind) mice. Proc. Natl. Acad. Sci. U S A 101, 13363-13367. doi: 10.1073/pnas.0403678101

Jin, K., Peel, A. L., Mao, X. O., Xie, L., Cottrell, B. A., Henshall, D. C., et al. (2004b). Increased hippocampal neurogenesis in Alzheimer's disease. Proc. Natl. Acad. Sci. U S A 101, 343-347. doi: 10.1073/pnas.2634794100

Jorstad, N. L., Wilken, M. S., Grimes, W. N., Wohl, S. G., VandenBosch, L. S., Yoshimatsu, T., et al. (2017). Stimulation of functional neuronal regeneration from Muller glia in adult mice. Nature 548, 103-107. doi: 10.1038/nature 23283

Karimi-Abdolrezaee, S., and Billakanti, R. (2012). Reactive astrogliosis after spinal cord injury-beneficial and detrimental effects. Mol. Neurobiol. 46, 251-264. doi: 10.1007/s12035-012-8287-4

Lang, B. T., Cregg, J. M., DePaul, M. A., Tran, A. P., Xu, K., Dyck, S. M., et al. (2015). Modulation of the proteoglycan receptor PTPsigma promotes recovery after spinal cord injury. Nature 518, 404-408. doi: 10.1038/nature13974

Li, H., and Chen, G. (2016). In vivo reprogramming for CNS repair: regenerating neurons from endogenous glial cells. Neuron 91, 728-738. doi: 10.1016/j. neuron.2016.08.004

Liu, M. H., Li, W., Zheng, J. J., Xu, Y. G., He, Q., and Chen, G. (2020). Differential neuronal reprogramming induced by NeuroD1 from astrocytes in grey matter versus white matter. Neural Regen. Res. 15, 342-351. doi: 10.4103/1673-5374. 265185

Liu, Y., Miao, Q., Yuan, J., Han, S., Zhang, P., Li, S., et al. (2015). Ascll converts dorsal midbrain astrocytes into functional neurons in vivo. J. Neurosci. 35, 9336-9355. doi: 10.1523/JNEUROSCI.3975-14.2015

Loeser, R. F. (2009). Aging and osteoarthritis: the role of chondrocyte senescence and aging changes in the cartilage matrix. Osteoarthritis Cartilage 17, 971-979. doi: 10.1016/j.joca.2009.03.002

Ma, T., Li, J., Xu, Y., Yu, C., Xu, T., Wang, H., et al. (2015). Atg5independent autophagy regulates mitochondrial clearance and is essential for iPSC reprogramming. Nat. Cell Biol. 17, 1379-1387. doi: 10.1038/ncb3256

Maryanovich, M., and Gross, A. (2013). A ROS rheostat for cell fate regulation. Trends Cell Biol. 23, 129-134. doi: 10.1016/j.tcb.2012.09.007

Matsuda, T., Irie, T., Katsurabayashi, S., Hayashi, Y., Nagai, T., Hamazaki, N., et al. (2019). Pioneer factor neurod1 rearranges transcriptional and epigenetic profiles to execute microglia-neuron conversion. Neuron 101, 472.e7-485.e7. doi: 10.1016/j.neuron.2018.12.010

Mattugini, N., Bocchi, R., Scheuss, V., Russo, G. L., Torper, O., Lao, C. L., et al. (2019). Inducing different neuronal subtypes from astrocytes in the injured mouse cerebral cortex. Neuron 103, 1086.e5-1095.e5. doi: 10.1016/j.neuron. 2019.08.009

Meletis, K., Barnabé-Heider, F., Carlén, M., Evergren, E., Tomilin, N., Shupliakov, O., et al. (2008). Spinal cord injury reveals multilineage differentiation of ependymal cells. PLoS Biol. 6:e182. doi: 10.1371/journal.pbio. 0060182

Mizushima, N., and Levine, B. (2010). Autophagy in mammalian development and differentiation. Nat. Cell Biol. 12, 823-830. doi: 10.1038/ncb0910-823

Mori, T., Tan, J., Arendash, G. W., Koyama, N., Nojima, Y., and Town, T. (2008). Overexpression of human S100B exacerbates brain damage and periinfarct gliosis after permanent focal ischemia. Stroke 39, 2114-2121. doi: 10.1161/strokeaha.107.503821

Mosteiro, L., Pantoja, C., Alcazar, N., Marión, R. M., Chondronasiou, D., Rovira, M., et al. (2016). Tissue damage and senescence provide critical signals for cellular reprogramming in vivo. Science 354:aaf4445. doi: 10.1126/science. aaf4445

Mosteiro, L., Pantoja, C., de Martino, A., and Serrano, M. (2018). Senescence promotes in vivo reprogramming through $\mathrm{p}^{\mathrm{INK} 4 a}$ and IL-6. Aging Cell 17:e12711. doi: 10.1111/acel.12711

Muthusamy, N., Brumm, A., Zhang, X., Carmichael, S. T., and Ghashghaei, H. T. (2018). Foxj1 expressing ependymal cells do not contribute new cells to sites of injury or stroke in the mouse forebrain. Mol. Psychiatry 8:1766. doi: 10.1038/s41598-018-19913-x

Niu, W., Zang, T., Smith, D. K., Vue, T. Y., Zou, Y., Bachoo, R., et al. (2015). SOX2 reprograms resident astrocytes into neural progenitors in the adult brain. Stem Cell Reports 4, 780-794. doi: 10.1016/j.stemcr.2015.03.006

Niu, W., Zang, T., Wang, L. L., Zou, Y., and Zhang, C. L. (2018). Phenotypic reprogramming of striatal neurons into dopaminergic neuron-like cells in the adult mouse brain. Stem Cell Reports 11, 1156-1170. doi: 10.1016/j.stemcr.2018. 09.004

Niu, W., Zang, T., Zou, Y., Fang, S., Smith, D. K., Bachoo, R., et al. (2013). In vivo reprogramming of astrocytes to neuroblasts in the adult brain. Nat. Cell Biol. 15, 1164-1175. doi: 10.1038/ncb2843

Obernier, K., Cebrian-Silla, A., Thomson, M., Parraguez, J. I., Anderson, R., Guinto, C., et al. (2018). Adult neurogenesis is sustained by symmetric self-renewal and differentiation. Cell Stem Cell 22, 221.e8-234.e8. doi: 10.1016/j.stem.2018.01.003

Okada, S., Nakamura, M., Katoh, H., Miyao, T., Shimazaki, T., Ishii, K., et al. (2006). Conditional ablation of Stat 3 or Socs 3 discloses a dual role for reactive astrocytes after spinal cord injury. Nat. Med. 12, 829-834. doi: 10.1038/nm1425

Osellame, L. D., Blacker, T. S., and Duchen, M. R. (2012). Cellular and molecular mechanisms of mitochondrial function. Best Pract. Res. Clin. Endocrinol. Metab. 26, 711-723. doi: 10.1016/j.beem.2012.05.003

Oyinbo, C. A. (2011). Secondary injury mechanisms in traumatic spinal cord injury: a nugget of this multiply cascade. Acta Neurobiol. Exp. 71, 281-299.

Pereira, M., Birtele, M., Shrigley, S., Benitez, J. A., Hedlund, E., Parmar, M., et al. (2017). Direct reprogramming of resident NG2 glia into neurons with properties of fast-spiking parvalbumin-containing interneurons. Stem Cell Reports 9, 742-751. doi: 10.1016/j.stemcr.2017.07.023

Pilz, G. A., Bottes, S., Betizeau, M., Jörg, D. J., Carta, S., Simons, B. D., et al. (2018). Live imaging of neurogenesis in the adult mouse hippocampus. Science 359, 658-662. doi: 10.1126/science.aao5056

Prieto, J., León, M., Ponsoda, X., Sendra, R., Bort, R., Ferrer-Lorente, R., et al. (2016). Early ERK1/2 activation promotes DRP1-dependent mitochondrial fission necessary for cell reprogramming. Nat. Commun. 7:11124. doi: $10.1038 /$ ncomms 11124

Prieto, J., and Torres, J. (2017). Mitochondrial dynamics: in cell reprogramming as it is in cancer. Stem Cells Int. 2017:8073721. doi: 10.1155/2017/8073721

Rasband, M. N. (2016). Glial contributions to neural function and disease. Mol. Cell. Proteomics 15, 355-361. doi: 10.1074/mcp.r115.053744

Ren, Y., Ao, Y., O’Shea, T. M., Burda, J. E., Bernstein, A. M., Brumm, A. J., et al. (2017). Ependymal cell contribution to scar formation after spinal cord injury is minimal, local and dependent on direct ependymal injury. Sci. Rep. 7:41122. doi: $10.1038 /$ srep41122 
Rivetti di Val Cervo, P., Romanov, R. A., Spigolon, G., Masini, D., MartínMontañez, E., Toledo, E. M., et al. (2017). Induction of functional dopamine neurons from human astrocytes in vitro and mouse astrocytes in a Parkinson's disease model. Nat. Biotechnol. 35, 444-452. doi: 10.1038/ nbt.3835

Robel, S., Berninger, B., and Götz, M. (2011). The stem cell potential of glia: lessons from reactive gliosis. Nat. Rev. Neurosci. 12, 88-104. doi: 10.1038/nrn2978

Sarsour, E. H., Kumar, M. G., Chaudhuri, L., Kalen, A. L., and Goswami, P. C. (2009). Redox control of the cell cycle in health and disease. Antioxid. Redox Signal. 11, 2985-3011. doi: 10.1089/ars.2009.2513

Shah, P. T., Stratton, J. A., Stykel, M. G., Abbasi, S., Sharma, S., Mayr, K. A., et al. (2018). Single-cell transcriptomics and fate mapping of ependymal cells reveals an absence of neural stem cell function. Cell 173, 1045.e9-1057.e9. doi: 10.1016/j.cell.2018.03.063

Smith, D. K., He, M., Zhang, C. L., and Zheng, J. C. (2017). The therapeutic potential of cell identity reprogramming for the treatment of aging-related neurodegenerative disorders. Prog. Neurobiol. 157, 212-229. doi: 10.1016/j. pneurobio.2016.01.006

Smith, D. K., and Zhang, C. L. (2015). Regeneration through reprogramming adult cell identity in vivo. Am. J. Pathol. 185, 2619-2628. doi: 10.1016/j.ajpath.2015. 02.025

Sofroniew, M. V. (2009). Molecular dissection of reactive astrogliosis and glial scar formation. Trends Neurosci. 32, 638-647. doi: 10.1016/j.tins.2009.08.002

Storer, M., Mas, A., Robert-Moreno, A., Pecoraro, M., Ortells, M. C., Di Giacomo, V., et al. (2013). Senescence is a developmental mechanism that contributes to embryonic growth and patterning. Cell 155, 1119-1130. doi: 10.1016/j.cell.2013.10.041

Su, M., Hu, H., Lee, Y., d'Azzo, A., Messing, A., and Brenner, M. (2004). Expression specificity of GFAP transgenes. Neurochem. Res. 29, 2075-2093. doi: 10.1007/s11064-004-6881-1

Su, Z., Niu, W., Liu, M. L., Zou, Y., and Zhang, C. L. (2014). In vivo conversion of astrocytes to neurons in the injured adult spinal cord. Nat. Commun. 5:3338. doi: $10.1038 /$ ncomms 4338

Tang, B. L. (2013). mTOR, autophagy and reprogramming. Front. Cell. Dev. Biol. 1:4. doi: $10.3389 /$ fcell.2013.00004

Torper, O., and Götz, M. (2017). Brain repair from intrinsic cell sources: turning reactive glia into neurons. Prog. Brain Res. 230, 69-97. doi: 10.1016/bs.pbr. 2016.12.010

Torper, O., Ottosson, D. R., Pereira, M., Lau, S., Cardoso, T., Grealish, S., et al. (2015). In vivo reprogramming of striatal NG2 glia into functional neurons that integrate into local host circuitry. Cell Rep. 12, 474-481. doi: 10.1016/j.celrep. 2015.06.040

Torper, O., Pfisterer, U., Wolf, D. A., Pereira, M., Lau, S., Jakobsson, J., et al. (2013). Generation of induced neurons via direct conversion in vivo. Proc. Natl. Acad. Sci. U S A 110, 7038-7043. doi: 10.1073/pnas.1303829110

Ueki, Y., Wilken, M. S., Cox, K. E., Chipman, L., Jorstad, N., Sternhagen, K., et al. (2015). Transgenic expression of the proneural transcription factor Ascl1 in Muller glia stimulates retinal regeneration in young mice. Proc. Natl. Acad. Sci. U S A 112, 13717-13722. doi: 10.1073/pnas.1510595112

Urrea, C., Castellanos, D. A., Sagen, J., Tsoulfas, P., Bramlett, H. M., and Dietrich, W. D. (2007). Widespread cellular proliferation and focal neurogenesis after traumatic brain injury in the rat. Restor. Neurol. Neurosci. $25,65-76$.

Wang, X., Gao, X., Michalski, S., Zhao, S., and Chen, J. (2016). Traumatic brain injury severity affects neurogenesis in adult mouse hippocampus. J. Neurotrauma 33, 721-733. doi: 10.1089/neu.2015.4097

Wang, C., Liang, C. C., Bian, Z. C., Zhu, Y., and Guan, J. L. (2013). FIP200 is required for maintenance and differentiation of postnatal neural stem cells. Nat. Neurosci. 16, 532-542. doi: 10.1038/nn.3365

Wang, L. L., Su, Z., Tai, W., Zou, Y., Xu, X. M., and Zhang, C. L. (2016). The p53 pathway controls SOX2-mediated reprogramming in the adult mouse spinal cord. Cell Rep. 17, 891-903. doi: 10.1016/j.celrep.2016.09.038

Wang, S., Xia, P., Rehm, M., and Fan, Z. (2015). Autophagy and cell reprogramming. Cell. Mol. Life Sci. 72, 1699-1713. doi: 10.1007/s00018-0141829-3
Wang, L. L., and Zhang, C. L. (2018). Engineering new neurons: in vivo reprogramming in mammalian brain and spinal cord. Cell Tissue Res. 371, 201-212. doi: 10.1007/s00441-017-2729-2

Weinberg, M. S., Criswell, H. E., Powell, S. K., Bhatt, A. P., and McCown, T. J. (2017). Viral vector reprogramming of adult resident striatal oligodendrocytes into functional neurons. Mol. Ther. 25, 928-934. doi: 10.1016/j.ymthe.2017. 01.016

Wilhelmsson, U., Li, L., Pekna, M., Berthold, C. H., Blom, S., Eliasson, C., et al. (2004). Absence of glial fibrillary acidic protein and vimentin prevents hypertrophy of astrocytic processes and improves post-traumatic regeneration. J. Neurosci. 24, 5016-5021. doi: 10.1523/JNEUROSCI.0820-04.2004

Winner, B., Regensburger, M., Schreglmann, S., Boyer, L., Prots, I., Rockenstein, E., et al. (2012). Role of $\alpha$-synuclein in adult neurogenesis and neuronal maturation in the dentate gyrus. J. Neurosci. 32, 16906-16916. doi: 10.1523/JNEUROSCI.2723-12.2012

Yamamoto, S., Yamamoto, N., Kitamura, T., Nakamura, K., and Nakafuku, M. (2001). Proliferation of parenchymal neural progenitors in response to injury in the adult rat spinal cord. Exp. Neurol. 172, 115-127. doi: 10.1006/exnr.2001. 7798

Yao, K., Qiu, S., Tian, L., Snider, W. D., Flannery, J. G., Schaffer, D. V., et al. (2016). Wnt regulates proliferation and neurogenic potential of muller glial cells via a Lin28/let-7 miRNA-dependent pathway in adult mammalian retinas. Cell Rep. 17, 165-178. doi: 10.1016/j.celrep.2016.08.078

Yao, K., Qiu, S., Wang, Y. V., Park, S. J. H., Mohns, E. J., Mehta, B., et al. (2018). Restoration of vision after de novo genesis of rod photoreceptors in mammalian retinas. Nature 560, 484-488. doi: 10.1038/s41586-018-0425-3

Yazdankhah, M., Farioli-Vecchioli, S., Tonchev, A. B., Stoykova, A., and Cecconi, F. (2014). The autophagy regulators Ambral and Beclin 1 are required for adult neurogenesis in the brain subventricular zone. Cell Death Dis. 5:e1403. doi: $10.1038 /$ cddis. 2014.358

Yokota, M., Hatakeyama, H., Okabe, S., Ono, Y., and Goto, Y. (2015). Mitochondrial respiratory dysfunction caused by a heteroplasmic mitochondrial DNA mutation blocks cellular reprogramming. Hum. Mol. Genet. 24, 4698-4709. doi: 10.1093/hmg/ddv201

Yoo, J., Lee, E., Kim, H. Y., Youn, D. H., Jung, J., Kim, H., et al. (2017). Electromagnetized gold nanoparticles mediate direct lineage reprogramming into induced dopamine neurons in vivo for Parkinson's disease therapy. Nat. Nanotechnol. 12, 1006-1014. doi: 10.1038/nnano.2017.133

Zhang, C., McNeil, E., Dressler, L., and Siman, R. (2007). Long-lasting impairment in hippocampal neurogenesis associated with amyloid deposition in a knock-in mouse model of familial Alzheimer's disease. Exp. Neurol. 204, 77-87. doi: 10.1016/j.expneurol.2006.09.018

Zhang, L., Lei, Z., Guo, Z., Pei, Z., Chen, Y., Zhang, F., et al. (2018). Reversing glial scar back to neural tissue through NeuroD1-mediated astrocyte-to-neuron conversion. bioRxiv [Preprint]. doi: 10.1101/261438

Zhao, C., Deng, W., and Gage, F. H. (2008). Mechanisms and functional implications of adult neurogenesis. Cell 132, 645-660. doi: 10.1016/j.cell.2008. 01.033

Zheng, W., ZhuGe, Q., Zhong, M., Chen, G., Shao, B., Wang, H., et al. (2013). Neurogenesis in adult human brain after traumatic brain injury. J. Neurotrauma 30, 1872-1880. doi: 10.1089/neu.2010.1579

Ziemka-Nałecz, M., and Zalewska, T. (2012). Endogenous neurogenesis induced by ischemic brain injury or neurodegenerative diseases in adults. Acta Neurobiol. Exp. 72, 309-324.

Conflict of Interest: The authors declare that the research was conducted in the absence of any commercial or financial relationships that could be construed as a potential conflict of interest.

Copyright (c) 2020 Tai, Xu and Zhang. This is an open-access article distributed under the terms of the Creative Commons Attribution License (CC BY). The use, distribution or reproduction in other forums is permitted, provided the original author(s) and the copyright owner(s) are credited and that the original publication in this journal is cited, in accordance with accepted academic practice. No use, distribution or reproduction is permitted which does not comply with these terms. 